\title{
Mixed model of stock price impact analysis and its application in bank stock
}

\author{
Weijia Kong ${ }^{1, ~ a}$, Mingrui Zhang, ${ }^{1, b}$, and Meijun Liu ${ }^{2, c}$ \\ ${ }^{1}$ Institute of Chemistry, Beijing Normal University, Haidian, Beijing, China \\ ${ }^{2}$ Department of Astronomy, Beijing Normal University, Haidian, Beijing, China \\ a hdblcc@126.com, ${ }^{\mathrm{b}}$ frokaikan@mail.bnu.edu.cn, ${ }^{\mathrm{c}} 1625831108 @ q q . c o m$
}

Keywords: ARMA model, stock price impact.

\begin{abstract}
With the rapid development of financial industry, financial automatic trading has gradually become the main business mode in today's social and economic activities. In the asset liquidation market, how to balance price shocks and time risks to ensure the lowest transaction cost is particularly important. In this paper, a reasonable mathematical model is established to fit the stock trend and describe the process of price change impact. The paper takes two stocks: Bank of China (SH601988) and Industrial and Commercial Bank of China (SH601398) as the example. The paper fits the stock trend by using ARMA model and describes the process of price change impact by using Nonlinear least squares regression model and improved ARMA model. The paper test the model by using actual data to test its successful rate, and sensitivity analysis. The result is satisfying.
\end{abstract}

\section{Introduction}

With the continuous improvement of the world economic development level, the financial industry has been developing rapidly, and a huge financial system has been established. The automatic trading system has many advantages: it can perform data analysis and calculation repeatedly and efficiently, and the ability of calculation and analysis is higher than that of human. At the same time, it can control the loss in a certain range because of its strict self-discipline. [1]

Due to the lack of autonomy, there is no mature system for computer automated transactions in asset liquidation transactions. If a suitable mathematical model is established, an asset clearing transaction algorithm is designed and the best trading strategy is provided to help the computer to trade, it can greatly reduce the transaction cost, increase the return on investment and the asset price, change the capital market structure, make the financial production flow more stable and sustainable. In order to achieve this goal, we hope that the price of assets will be influenced by the asset transaction behaviour itself and the time risk, to establish a relatively best mathematical model of the fitting effect, and to describe the process of price change. By analyzing the trading data of two shares of BOC and ICBC in 2017, we describe the impact of the change in the supply and demand on the transaction assets caused by the huge trading of institutional investors and the impact on the price, and describe the price shocks. Then by considering the influence of time and cost, the model is established to analyze the selling forecast under different strategies, and the optimal transaction strategy is calculated respectively.

\section{Present research situation}

In investment, we often need to predict the fluctuation of stock price over time, so as to avoid potential risks. In this regard, Robert. Engle[2] put forward the ARCH model in 1982 to predict the trend of time series. Bollerslev[3] combined the ARMA model with the ARCH model in 1986, and proposed the GARCH model, which greatly simplifies the complexity of the ARCH model. Brennan and other [4] think that we can estimate the volume of the next transaction according to the volume and transaction price of the previous transactions. Craig[5] thinks that buying and selling can be modeled by GARCH model. These studies show that the ARMA and GARCH models are effective for stock price changes over time. Chang[7] used the VAR model to predict investment in 
bank stocks. On the basis of predecessors' research, this paper will analyze bank stock with ARMA and GARCH model, and describe the price impact on the basis of literature, so as to facilitate the analysis of asset liquidation and income.

\section{Description the process of price change based on ARMA model}

Stock is a typical time series data. A mathematical model can be established by time series analysis by curve fitting and parameter estimation. Time series analysis can be used to objectively describe, analyze and predict the future, decision-making and control of the system. We use the ARMA model to describe the price change process under the influence of asset price change and time risk.

\subsection{Sequential analyses}

Define the imported time series data as sequence $P$, and establish the logarithmic yield sequence $r$ by the following formula:

$r=d \log (p)$

Table 1 The result of logarithmic yield sequence

\begin{tabular}{|c|c|c|c|c|c|}
\hline & Mean & Skewness & Kurtosis & Jarque-Bera & Probability \\
\hline$I C B C$ & $3.69 * 10^{\wedge}-6$ & -6.378777 & 518.4090 & $6.97 * 10 \wedge 8$ & 0.0 \\
\hline$B O C$ & $2.20 * 10^{\wedge}-6$ & -0.675793 & 68.49775 & $1.12 * 10^{\wedge} 7$ & 0.0 \\
\hline
\end{tabular}

According to the analysis of histogram, the logarithmic yield sequence skewness of the two graph is less than 0 , and the kurtosis is far higher than the kurtosis of the Yu Zheng state, indicating that the yield sequence does not have the characteristics of peak and tailing. If the $P$ value is 0 , we reject the hypothesis that the logarithmic yield sequence obeys normal distribution. We can see that the data concentration is very high and does not obey normal distribution.

Through the analysis of the autocorrelation function analysis of the logarithmic return rate, we can see that the corresponding $\mathrm{p}$ value of $\mathrm{Q}$ statistics is close to 0 , so there is a significant correlation in the sequence.

\subsection{Arma model}

\subsubsection{Description}

The ARMA model is a more mature model for short-term prediction, which requires a large number of data and requires the data to be random and smooth, and these two stocks meet these conditions.

1) The AR model is also called the autoregressive model, which uses the past observation values and the current interference values to carry out the linear combination of the prediction process. The mathematical formula of this model is:

$$
Y_{t}=\sum_{i=1}^{p} H_{i} Y_{t-i}+e_{t}
$$

In the formula, $\mathrm{P}$ is the order of autoregressive models; $\mathrm{Hi}$ ( $\mathrm{i}=1,2, \ldots \mathrm{P}$ is the undetermined coefficient of the model; et is the error; Yt is a time series.

2) The MA model, also called the sliding average model, uses the past interference values and the current interference values to perform the linear combination of the prediction process. The mathematical formula of the model is:

$$
Y_{t}=e_{t}-\sum_{i-1}^{q} \theta_{i} e_{t-i}
$$

In the formula: $q$ is the order of the model; $\theta j(j=1,2, \ldots)$ is the undetermined coefficient of the 
model; et is the error; Yt is the observed value.

3) The ARMA model, combines the above two models and can be used to describe the stochastic stationary process. The mathematical formula of the model is:

$$
Y_{t}=\left(\sum_{i=1}^{p} H_{i} Y_{t-i}\right)+\left(e_{t}-\sum_{i=1}^{q} \theta_{i} e_{t-i}\right)
$$

The order of the model can be determined by examining the autocorrelation function and partial correlation function of the variables.

For the AR model, the partial correlation function Hki is truncated. For the P order AR model, the partial correlation function $\mathrm{Hki}=0(\mathrm{k}>\mathrm{p})$ is obtained. When the $\mathrm{Hk}=0$ is obtained, the order of the model can be determined to be $\mathrm{k}-1$.

For the MA model, the autocorrelation function DK is truncated. For the Q order MA model, its autocorrelation function $\mathrm{dk}=0(\mathrm{k}>\mathrm{q})$. If $\mathrm{dk}=0$ is obtained, the order of the model can be determined to be $\mathrm{k}-1$.

\subsubsection{Parameter estimation}

Then the least square method is used to estimate the parameters

$$
Y=(X+E) U
$$

In the formula: $\mathrm{Y}$ is the observed value now; $\mathrm{X}$ is the historical observation value; $\mathrm{U}$ is the error. On the estimation of $U$, build the quation:

$$
Q=(Y-X U)^{*}(Y-X U)=U^{*} U
$$

$\mathrm{Q}$ is called the sum of squares of errors, so that the minimum $\mathrm{Q}$ value of $\mathrm{U}$ is called the least squares estimate of $U$. Using the first derivative of the mathematical formula, $U$ obtains the unique solution: $U=X^{*}(X-l Y)$.

\subsubsection{Simulation results}

By observing the autocorrelation and partial autocorrelation coefficients and their graphs of two stocks, we initially determined the moving average order $\mathrm{q}=1$ and autoregressive coefficient $\mathrm{p}=1$. So we use the ARMA $(1,1)$ model to simulate:

The simulation results of ARMA $(1,1)$ model of Bank of China are as follows:

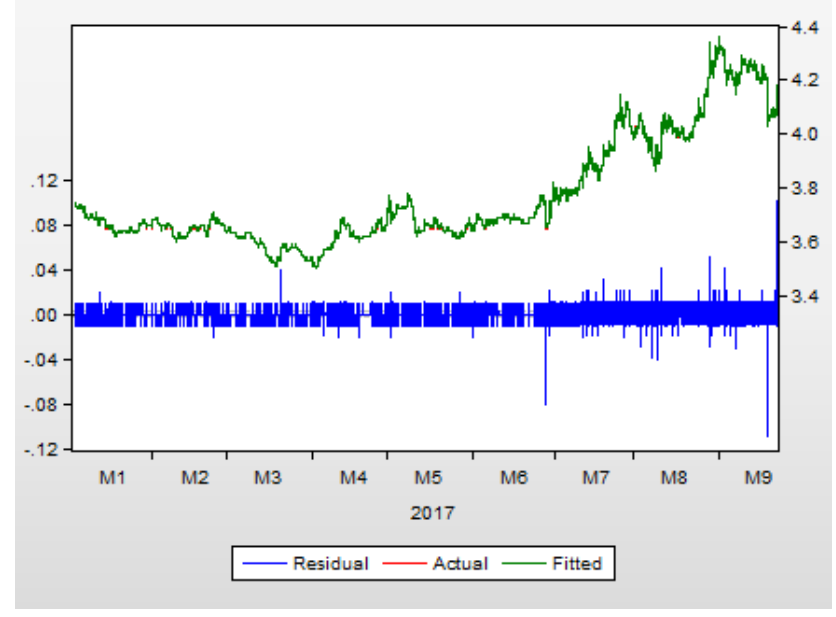

Figure 1 ARMA result of BOC

The equation of the price fitting of the Bank of China is:

$$
M_{t}=3.867+1.000 M_{t-1}-0.1641 T_{t-1}+T_{t}+\varepsilon_{t}
$$


Among them, Mt represents the trading price at $\mathrm{t}$ time, $\mathrm{Tt}$ is the time of $\mathrm{T}$, and $\mathrm{t}$ is noise.

The R-squared of the prediction equation reaches 0.9998, and the fitting effect is satisfactory. Therefore, the above trading price prediction formula and the right picture can be used to describe the price change process of Bank of China.

The simulation results of ARMA $(1,1)$ model of Industrial and Commercial Bank of China are as follows:

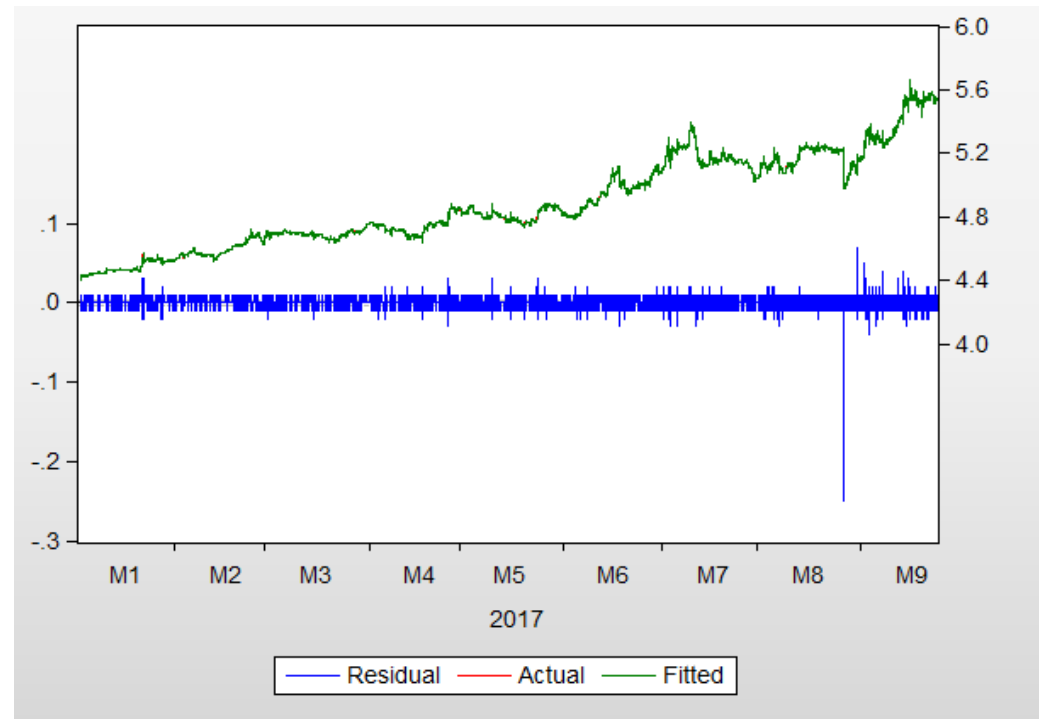

Figure 2 ARMA result of ICBC

The equation of the price fitting of ICBC is:

$$
M_{t}=5.292+1.000 M_{t-1}-0.004316 T_{t-1}+T_{t}+\varepsilon_{t}
$$

The R-squared of the prediction equation reaches 0.9999 , the fitting effect is satisfactory as well.

\section{The estimate of the stock price impact phenomenon}

\subsection{Nonlinear least squares regression estimation}

The traditional hypothesis based on the impact of the stock change price is mostly based on the intuition impression that with the increase of the present quantity, the stock price will fall at a rate of positive ratio to the variable present quantity, although it can simplify the model, but it can not accurately describe the actual situation. In this paper, we use nonlinear least squares regression to estimate the price shock function PI of stock realisation.

The parameters $\mathrm{X}$ and $\mathrm{y}$ are defined as follows:

$$
\begin{gathered}
x=\frac{Q_{t+1}-Q_{t}}{Q_{t}} \\
y=\frac{v_{t}}{10000}
\end{gathered}
$$

Among them, Qt is the highest bid price of $\mathrm{T}$, the transaction amount at $\mathrm{t}$ time. Then the transaction price $\mathrm{M}$ at $\mathrm{t}$ time can be expressed as:

$$
M_{t}=a x+\frac{b\left(y^{c}-1\right)}{c}+d b+\lambda_{y}+\varepsilon \lg t+R
$$

The final price impact function is the difference of $\mathrm{M}$ :

$$
P I=d \log M
$$


For the Bank of China's stock market, a nonlinear model of price impact is constructed by fitting the above models.

$$
P I=3.77279-15.2132 x-0.0174487\left(y^{0.607798}-1\right)+0.0219241 *(\text { noise })+0.004106 y+0.009896 \lg t
$$

For the shares of ICBC, we can also draw the equation:

$$
P I=5.08781-26.909 x-0.0699819\left(y^{0.5186}-1\right)-0.0407058^{*}(\text { noise })+0.002858 y+0.003873 \lg t
$$

\subsection{Description of price shocks based on ARMA model}

When building ARMA models based on price shocks, we take into account the impact of price shocks on the maximum bid price and transaction volume. By comparing the autocorrelation coefficient and the partial autocorrelation coefficient and the $t$ test results of several different models, we determine the order of the model and use the ARMA $(1,6)$ model to simulate the price impact.

The price impact function is:

$$
P I=d \log M_{t}
$$

The forecast value of the change of the stock price of the Bank of China can be obtained as follows:

$$
M_{t}=-4.5916 * 10^{-5} y_{t}+1.9046 x+1.000 M_{t-1}+0.1034 T_{t-6}+T_{t}+\varepsilon_{t}
$$

The R-squared is 0.9830 . Therefore, we can use the above price impact function to describe the price impact of Bank of China.

The forecast value of the change of the stock price of ICBC can be obtained as follows:

$$
M_{t}=-3.199 * 10^{-5} y_{t}+1.3207 x+1.000 M_{t-1}-0.05701 T_{t-6}+T_{t}+\varepsilon_{t}
$$

The R-squared is 0.9915 . Therefore, we can use the above price impact function to describe the price impact of ICBC shares.

\section{Model test}

Take the transaction data of the first 8 months into the ARMA model, predict the transaction data for ninth months, and draw the similarity test.

For the BOC stock model and the ICBC stock model, the detection similarity is $92.7274 \%$ and $84.6846 \%$ respectively.

The overall detection effect is good, and the model can be used to predict transaction data as well.

The prediction of the price of the transaction is added to a perturbation (using the random function plus a small perturbation within 0.01 ), which is used to calculate the predicted volume of transaction, and the forecast transaction volume and the forecast transaction price are very clear.

There is a significant negative correlation; according to the calculation, about $140 \%-160 \%$ of price changes will be reflected in the volume of transactions.

In summary, the analysis shows that the ARMA model in this problem will have an impact on the small fluctuations, and the sensitivity is high.

\section{Conclusion}

The time series of our two bank stocks is stable and the residuals are very small, which fully comply with the ARMA model's construction principle. By establishing a suitable ARMA model, we get a reasonable description of the stock trend rule. In describing the price impact, we first used the nonlinear least squares regression, and then used the ARMA model. Compared with the original ARMA model, we introduced the actual impact factor $\mathrm{x}, \mathrm{y}$ to describe the price shock more practical. The model test results are satisfying and the sensitivity is high. Since the amount of test data is large, the result is convincible. 


\section{References}

[1] Minghui Xun, Guifeng Shi. GARCH model of mixed distribution information of stock price impact and its application [J]. Journal of Shanghai Jiao Tong University,2007(07):1105-1109.

[2] Engle R F. Autoregressive Conditional heteroscedasticity with estimates of the variance of United Kingdom inflation. Econometrica, 50, 987-1008.

[3] Bollerslev T. Generalized autoregressive conditional heteroscedasticity. Journal of Econometrics, 31, 307-27

[4] Glostern L R, Harris L E. Estimating hte components of bidask spread [J]. Journal of Financial Economics, 1988, 21: 132-142.

[5] Bernnan M J, Avanidhar S. Marker microstructure and asset princing: On the compensation of illiquidity in stock returns [J]. Journal of Financial Economics 1996, 41: 441-464.

[6] Craig A D. Good news, bad news and GARCH effect in stock return data [J]. Journal of Applied Economics, 2001, 11: 313-327.

[7] Changze Wei. A+H research on the linkage of bank shares [D]. Huaqiao University,2013. 